\title{
The Functions of Nonsuicidal Self-Injury: Support for Cognitive- Affective Regulation and Opponent Processes From a Novel Psychophysiological Paradigm
}

\author{
Joseph C. Franklin, Elenda T. Hessel, Rachel V. Aaron, Michael S. Arthur, Nicole Heilbron, \\ and Mitchell J. Prinstein \\ University of North Carolina at Chapel Hill
}

\begin{abstract}
Although research on the reasons for engaging in nonsuicidal self-injury (NSSI) has increased dramatically in the last few years, there are still many aspects of this pernicious behavior that are not well understood. The purpose of this study was to address these gaps in the literature, with a particular focus on investigating whether NSSI (a) regulates affective valence in addition to affective arousal and (b) serves a cognitive regulation function in addition to an affect regulation function. To elucidate these issues, the present study utilized a sample of 112 participants (33 controls, 39 no pain controls, 16 NSSI individuals, and 24 controls matching the affect dysregulation levels of the NSSI group), employed psychophysiological measures of affective valence (startle-alone reactivity) and quality of information processing (prepulse inhibition), and used experimental methods involving an NSSI-proxy to model the NSSI process. Results largely were consistent with predictions, supporting the hypotheses that NSSI serves to regulate cognitive processing and affective valence. On this latter point, however, the control groups also showed a decrease in negative affective valence after the NSSI-proxy. This unexpected finding is consistent with the hypothesis that opponent processes may contribute to the development of self-injurious behaviors (Joiner, 2005). Overall, the present study represents an important extension of previous laboratory NSSI studies and provides a fertile foundation for future studies aimed at understanding why people engage in NSSI.
\end{abstract}

\section{Keywords}

nonsuicidal self-injury; NSSI; prepulse inhibition; PPI; affect dysregulation

\begin{abstract}
Nonsuicidal self-injury (NSSI) is defined as the direct and intentional destruction of one's own body tissue in the absence of suicidal intent (Nock, Joiner, Gordon, Lloyd-Richardson, \& Prinstein, 2006; Nock \& Prinstein, 2004). Reports of the prevalence of NSSI vary
\end{abstract}

\footnotetext{
(C) 2010 American Psychological Association

Correspondence concerning this article should be addressed to Joseph C. Franklin, Department of Psychology, University of North Carolina at Chapel Hill, CB\#3270 Davie Hall, Chapel Hill, NC 27599-3270. franjc1@email.unc.edu.

Joseph C. Franklin, Elenda T. Hessel, Rachel V. Aaron, Michael S. Arthur, Nicole Heilbron, and Mitchell J. Prinstein, Department of Psychology, University of North Carolina at Chapel Hill.

Elenda T. Hessel is now at the Department of Psychology, University of Virginia, Charlottesville. Rachel V. Aaron is now at the

Department of Psychology, Vanderbilt University.
} 
according to the population studied and severity of NSSI behaviors meeting inclusion criteria; however, studies have reported a prevalence of $17 \%$ in college populations (Whitlock, Eckenrode, \& Silverman, 2006) and 40\%-60\% in adolescent clinical populations (Darche, 1990; DiClemente, Ponton, \& Hartley, 1991). In addition to being a health-risk behavior in its own right, NSSI confers an increased risk of suicidal self-injury (Joiner, 2005; Nock et al., 2006). Despite an informative and burgeoning literature on the functions of NSSI, there is still much to be learned about this phenomenon. The purpose of the present study was to provide a greater understanding of why individuals engage in NSSI by addressing several gaps in the current literature.

In a recent review of 18 published studies on the functions of NSSI, Klonsky (2007) determined that this literature was nearly unanimous in finding that (a) acute negative affect precedes NSSI and (b) after NSSI, there is a sense of relief and a decrease in negative affect (i.e., an automatic negative reinforcement [ANR] function; cf. Nock \& Prinstein, 2004). It should be noted that Klonsky (2007) also found moderate support for self-punishment and automatic positive reinforcement (APR) functions. This literature is comprised primarily of studies that utilized correlational designs and self-report methods. Such studies have provided a valuable foundation for the study of NSSI and continue to yield crucial information; nevertheless, they have a limited ability to determine causality and are vulnerable to self-report biases. As articulated by Janis and Nock (2009), "the mental processes involved in thinking about, planning, and enacting NSSI may be outside of conscious awareness. People are much more capable of accurately reporting that they have engaged in a specific behavior; however, it is less likely that they can accurately explain the cognitive processes underlying this behavior" (p. 261). Indeed, Janis and Nock found that whereas NSSI was associated with greater self-reported impulsivity, it was not associated with greater impulsivity on performance-based measures.

Laboratory studies (i.e., experimental and/or psychophysiological studies) may accordingly provide important information about the functions of NSSI. Five laboratory NSSI studies have been conducted, with each measuring self-report and/or psychophysiological variables before and after the administration of an NSSI-proxy (i.e., an experimental stimulus designed to approximate some aspect of NSSI such as pain). The results of these studies have generally been interpreted as supporting the ANR function of NSSI (Klonsky, 2007). Russ et al. (1992) used a cold pressor test (CPT) as an NSSI-proxy. In a group of borderline personality disorder (BPD) patients who reported feeling no pain during self-injury, results showed that self-reported levels of anger, depression, anxiety, and confusion were decreased after the NSSI-proxy. In a similar study, Schmahl et al. (2006) used thermode-generated heat stimuli as an NSSI-proxy and measured brain activity with blood oxygen leveldependent functional magnetic resonance imaging (BOLD fMRI) throughout the experiment. Results revealed that, during administration of painful heat stimuli, a sample of BPD patients showed greater dorsolateral prefrontal cortex activity and decreased amygdala and anterior cingulate cortex activity relative to nonpatient controls. The authors concluded that this neural pattern represents an antinociceptive mechanism that works by downregulating the emotional components of pain in BPD patients with a history of NSSI. These are the only two published studies that have used a painful stimulus as an NSSI-proxy. The three other laboratory studies utilized NSSI imagery scripts as their NSSI-proxies and 
measured autonomic variables (e.g., skin conductance, heart rate) in samples of incarcerated men (Haines, Williams, Brain, \& Wilson, 1995), women diagnosed with BPD (Welch, Linehan, Sylvers, Chittams, \& Rizvi, 2008), and a more general psychiatric sample (Brain, Haines, \& Williams, 1998). These three studies generally found a reduction in autonomic activity postproxy in the NSSI groups. Taken together, the five extant laboratory NSSI function studies provide important support for the ANR function across multiple psychiatric populations, several psychophysiological measures, and a variety of NSSI-proxies.

These studies represent important strides toward understanding the functions of NSSI; however, there is still much to be learned about NSSI. First, given the high prevalence rate of NSSI in psychiatric and incarcerated populations, it makes sense that the extant laboratory studies utilized psychiatric samples. Nevertheless, NSSI is also common in nonclinical populations (see e.g., Whitlock et al., 2006), and it is unclear whether these findings would generalize to a nonclinical sample, among which the frequency of self-injury and degree of affect dysregulation would likely be decreased. To address this, the present study employs a nonclinical sample. Second, because affect dysregulation is associated with both abnormal pain processing (see Klossika et al., 2006) and NSSI (Gratz \& Roemer, 2008), it is possible that affect dysregulation differences between control and NSSI groups, rather than a history of NSSI, explains the abnormal pain processing in these studies. The present study controls for this possible confound by including a control group that matches the affect dysregulation levels of the NSSI group. Third, given that NSSI almost always occurs in the context of acute negative affect (Klonsky, 2007), the ecological validity of previous studies has been limited in that they have not induced acute negative affect before administering the NSSI-proxy. The present study accordingly induces negative affect before the NSSI-proxy. Thus, it is possible to experimentally examine how a painful stimulus is associated with cognitive and affective regulation following stress-induced dysregulation.

Fourth, perhaps one of the biggest limitations of the NSSI function literature is that previous studies have employed only measures of self-reported affect and/or measures of physiological arousal; none of these studies utilized an independent measure of affective valence (though one nonlaboratory study has examined this; Klonsky, 2009). This is important because affect is composed of both arousal (the intensity of affect) and valence (positive or negative tone of affect; Lang, Bradley, \& Cuthbert, 1990). Accordingly, a decrease in physiological arousal does not necessarily indicate a less negatively valenced affective state. Similarly, a reduction in self-reported negative affect could be explained by a decrease in arousal. Thus, it is not clear whether (a) NSSI only serves to reduce the intensity of affect or (b) it also serves to reduce negative affective valence. In other words, does NSSI just reduce the intensity of what one is feeling, or does it help one feel better? Resolving this issue would help to clarify the affective function of NSSI.

Perhaps the most popular and best validated psychophysiological measure of affective valence is the acoustic startle reflex, a defensive response that occurs in reaction to a sufficiently sudden and intense sound (Blumenthal et al., 2005). The startle response is mediated by a nucleus in the brain stem, the nucleus reticularis pontis caudalis, which receives modulatory input from the respective areas of the brain associated with positive and negative affect (i.e., the limbic areas; Koch \& Fendt, 2003). The motivational priming 
hypothesis (Lang et al., 1990) states that because the startle response is a defensive reaction and thus has a negative valence, stimuli that match this negative valence cause phasic increases in startle reactivity relative to neutral stimuli. Correspondingly, stimuli that do not match this negative valence cause phasic decreases in startle reactivity relative to neutral stimuli (for reviews see Bradley, Cuthbert, \& Lang, 1999; Lang et al., 1990). Startle has been employed extensively to investigate affective valence in internalizing (Vaidyanathan, Patrick, \& Cuthbert, 2009), externalizing (see e.g., Patrick, Bradley, \& Lang, 1993), and psychotic (see e.g., Yee et al., 2010) disorders. On the basis of the hypothesis that-in addition to reducing arousal-NSSI decreases negative valence, we propose that individuals with a history of NSSI should show decreased startle after the NSSI-proxy, whereas controls should show increased startle.

Fifth, although studies of the affective functions of NSSI are important, the laboratory NSSI literature has heretofore overlooked possible concurrent functions of NSSI, such as a cognitive regulation function. Specifically, affect dysregulation may be concomitant with disrupted information processing, representing a neural system that is biased toward affectrelevant information and action, thereby decreasing problem-solving skills and leading to more impulsive and maladaptive behaviors such as NSSI. In terms of dialectical behavior therapy (Linehan, 1993), individuals may engage in NSSI when they are in emotion mind, and NSSI may be associated with a shift toward reasonable mind. We hypothesize a cognitive regulation function based in part on evidence suggesting that affective and cognitive regulation may be ontologically indistinguishable (cf. Duncan \& Barrett, 2007). Because affective and cognitive regulation should be phenomenologically distinguishable (cf. Duncan \& Barrett, 2007), however, we contend that affective and cognitive regulation are not redundant. Affective regulation should be associated with feeling better, whereas cognitive regulation should be associated with thinking more clearly. Unfortunately, the cognitive regulation function has not been directly investigated, in part because there are few direct psychophysiological measures of information processing that could be employed in an NSSI laboratory study.

Fortunately, another startle modulation measure-prepulse inhibition (PPI) of acoustic startle-is a measure of the quality of information processing that could be employed in laboratory NSSI studies. PPI occurs when a stimulus (i.e., the prepulse) is presented 30-500 ms before a startle-eliciting stimulus and causes decreased startle reactivity to the startle stimulus relative to nonprepulse trials (Blumenthal, 1999). PPI is mediated by an area of the midbrain called the pedunculopontine tegmental nucleus (PPTg), which receives modulatory input from neural circuits associated with cognitive processing (e.g., the basal forebrain, mesocortical dopamine system, the thalamus) and arousal (e.g., the mesolimbic dopamine system; Swerdlow, Geyer, \& Braff, 2001; Winn, 2006). PPI occurs because when the prepulse reaches the PPTg, the PPTg uses its wide-ranging influence in the brain to decrease neurological noise (e.g., by temporarily blocking incoming sensory information such as startle and by inhibiting thalamic activity not directly due to the prepulse) and to increase neurological processing relevant to the prepulse (e.g., by activating specific dopaminergic, thalamic, and cortical areas). Thus, PPI reflects the state of information processing in the brain, with greater cognitive processing being associated with higher PPI. 
The construct validity of PPI as an index of cognitive processing is supported by the fact that greater executive functioning (Bitsios \& Giakoumaki, 2005; Bitsios, Giakoumaki, Theou, \& Frangou, 2006) and increased attention to the prepulse (Dawson, Schell, Hazlett, Nuechterlein, \& Filion, 2000) are associated with increased PPI. In contrast, populations associated with poorer executive functioning (see e.g., Braff et al., 1978; Cadenhead, Geyer, \& Braff, 1993) and increased arousal (see e.g., Duley, Hillman, Coombes, \& Janelle, 2007; Franklin, Bowker, \& Blumenthal, 2009; Grillon \& Davis, 1997) are associated with lower PPI at baseline. Because NSSI is associated with affect dysregulation and impulsivity (and thus poorer cognitive processing; see Duncan \& Barrett, 2007), individuals who engage in NSSI should display decreased PPI at baseline, and all groups should display decreased PPI during a stressor test (cf. Grillon \& Davis, 1997). Additionally, if NSSI serves a cognitive regulation function, individuals with a history of NSSI should display an increase in PPI after an NSSI-proxy, whereas individuals without a history of NSSI should display a decrease in PPI.

In summary, the present study addresses several important questions about the functions of NSSI — both methodological and conceptual. Perhaps most importantly, the present study is the first to investigate the hypotheses that NSSI serves to regulate affective valence and cognitive processing.

\section{Method}

\section{Participants}

Participants were 112 young adults: 77 female, 35 male; 83.90\% European American, 8.90\% Asian American, 5.36\% African American, and 1.79\% Hispanic American; ranging in age from $18-29$ years $(M=19.73, S D=1.62)$. Participants were recruited from two sources: (a) introductory psychology classes that included a research participation option ( $n$ $=77$ ) and (b) campus-wide e-mail advertisements that offered payment of $\$ 20$ for participation in the study $(n=35)$. Participants recruited from e-mail advertisements were recruited on the basis of either (a) their high affect dysregulation or NSSI scores on our screening questionnaire, which they completed as part of a separate experiment during a summer college orientation program $(n=26)$, or (b) their affirmative response to a campuswide e-mail that asked, "In the last year, have you purposefully injured yourself without intending to die (e.g., cutting or burning your skin) more than 6 times?" $(n=9)$.

Participants were sorted into the following four groups on the basis of their responses on NSSI and affect dysregulation questionnaires: low affect dysregulation with no history of NSSI (control group; $n=33$ ), high affect dysregulation with no history of NSSI (matched affect dysregulation [matched-AD] group; $n=24)$, no history of NSSI and not given a painful stimulus during the experiment (no pain group; $n=39$ ), and history of NSSI (NSSI group; $n=16$; see Table 1 for NSSI descriptives). Participants scoring above the overall median on the Difficulties in Emotion Regulation Scale (DERS; Gratz \& Roemer, 2004) were placed in the matched-AD group; participants scoring below the overall median were placed in the control group. Given that we employed a college population, the purpose of the matched-AD group was not to provide a group that was experiencing clinical levels of affect dysregulation but rather to provide a stringent control group that approximated the level of 
affect dysregulation in the NSSI group. The matched-AD group thus allowed us to examine whether an NSSI history per se, and not merely elevated affect dysregulation, was associated with abnormal responses to pain in the experimental paradigm. A one-way analysis of variance revealed that there was a significant effect of group on DERS total scores, $F(3$, $111)=25.33, p<.001$, with a post hoc Tukey's honestly significant difference test indicating that the NSSI $(M=87.13, S D=14.16)$ and matched-AD $(M=86.96, S D=14.39)$ groups reported comparable levels of affect dysregulation. Moreover, both groups reported significantly higher levels of affect dysregulation than did the control $(M=62.53, S D=$ $10.94)$ and no pain $(M=66.67, S D=13.52)$ groups $(p s<.001)$. Chi-square analyses revealed that gender, age, and ethnicity did not differ by group (all $p \mathrm{~s}>.05$ ), and univariate analyses showed that there were no gender, age, or ethnicity effects on psychophysiological variables (all $p s>.05)$.

\section{Procedure}

Screening-Participants recruited from introductory psychology classes entered the study without being screened; subsequently, these participants filled out the screening questionnaire (to be consistent with the other groups), the DERS, and the Functional Assessment of Self-Mutilation (FASM; Lloyd-Richardson, 2008) and were sorted into one of the four groups. Participants recruited through other means (see previous section) were screened on the basis of an abbreviated version of the DERS and a one-item NSSI question. Pending their responses on these questionnaires, participants were recruited into the study, at which time they took the full DERS and the FASM. As with the introductory psychology participants, these participants were grouped on the basis of their responses on the full DERS and FASM.

Experimental session-Participants were seated individually in a sound-attenuated room, where they read and signed an informed consent form. After this, participants filled out the battery of questionnaires. After completing these measures, participants underwent the following preparations for the startle portion of the experiment: (a) The skin on the left temple and below the left eye was cleaned with a cotton swab dipped in rubbing alcohol; (b) surface recording electrodes filled with Signa Electrode Cream (Parker Laboratories) were then placed on the cleaned areas-one electrode was attached to the skin overlaying the orbicularis oculi muscle directly below the pupil but below the lower eyelid, another electrode was placed approximately $15 \mathrm{~mm}$ (center to center) lateral to and slightly higher than the other electrode, and the ground electrode was placed on the skin overlaying the left temple; and (c) headphones then were placed comfortably on the participant (cf. Blumenthal et al., 2005; Blumenthal, Noto, Fox, \& Franklin, 2006).

Background noise was then turned on (and remained on throughout the session), and participants were given $3 \mathrm{~min}$ to acclimate to it before any other stimuli were presented. Following standard procedure, three habituation trials of a $100-\mathrm{dB}(\mathrm{A})$ startle stimulus were then presented (these trials are not included in analyses). Next, two sets of trial blocks were presented (see Figure 1): Set A was composed of three blocks (i.e., baseline, first stressor, first administration of the NSSI-proxy), and Set B was composed of two blocks (i.e., second stressor and second administration of the NSSI-proxy). Each block was composed of 12 
trials, with each trial containing a $100-\mathrm{dB}(\mathrm{A})$ startle stimulus, and half of the trials also containing an $85-\mathrm{dB}(\mathrm{A})$ prepulse, resulting in six startle-alone and six PPI trials per condition. Within blocks, trial order varied randomly. Each block was followed by a 3-min period during which the participant either (a) rested and was given instructions for a speech task (described later in the Stimuli section) or (b) underwent the NSSI-proxy, which was the CPT (also described in the Stimuli section). Subjective units of distress (SUDS) were assessed at five points during the experiment, each corresponding with these psychophysiological measurement points: (a) before the baseline block, (b) immediately after the first stress block, (c) immediately after the first CPT, (d) immediately after the second stress block, and (e) immediately after the second CPT.

Block 1 was the baseline block. Following this, participants rested for 3 min and then were given instructions for one of their two speech tasks (the speech prompt order was counterbalanced across participants). Participants then were given 4 min to prepare for this speech, during which time Block 2 (i.e., the Stress 1 block) was presented. Next, the CPT was administered (the hand order was counterbalanced both within and across participants); the CPT lasted for a maximum of $2 \mathrm{~min}$ (the preparation for, and execution of, the CPT lasted approximately $4 \mathrm{~min}$ for each participant). Immediately upon completion of the CPT, Block 3 (the Pain 1 block) was administered. Participants then rested for 3 min and, subsequently, were given instructions for their second speech task. Block 4 (the Stress 2 block) was presented during the 4-min preparation interval for this speech. Finally, the CPT was administered again (to the opposite hand), immediately after which Block 5 (the Pain 2 block) was presented. Participants were then debriefed, compensated, and allowed to leave. The aforementioned procedure applied to the NSSI, matched-AD, and control groups; the no pain group followed the same procedure minus the administration of the CPT (i.e., instead of the CPT, participants rested before Blocks 3 and 5).

\section{Stimuli}

Acoustic stimuli-All stimuli settings were in accordance with recommendations from parametrical PPI studies (Blumenthal et al., 2005; Franklin et al., 2009). Startle stimuli were $100-\mathrm{dB}$ (A) broadband noises $(20 \mathrm{~Hz}-20 \mathrm{kHz})$, with a 50-ms duration and a rise/fall time of $<1 \mathrm{~ms}$. Prepulses were $85-\mathrm{dB}(\mathrm{A})$ broadband noises, each with a $40-\mathrm{ms}$ duration and a rise/ fall time of $<1 \mathrm{~ms}$. The stimulus onset asynchrony (prepulse to startle stimulus) for each trial was $120 \mathrm{~ms}$. Background noise was a continuous 70-dB(A) broadband noise present during the entire testing session. Intertrial intervals varied randomly from $14 \mathrm{~s}$ to $23 \mathrm{~s}$. All stimuli were generated by Adobe Audition, presented by SuperLab, and delivered to the participants through Sennheiser PX200 headphones. Stimulus intensities were calibrated with steadystate signals presented through the headphones and measured with a sound level meter.

Speech tasks-Participants in this study were given two speech tasks. For one, the topic was "Give a speech about why you should be picked to be on a reality show about people your age," and for the other, the topic was "Give a speech about whether or not you believe it is right for the government to execute people." Participants were given 4 min to prepare for, and 1 min to deliver, each speech. Participants delivered their speeches in front of a video camera and a monitor that displayed their live image. Additionally, participants were 
told that their speech would be recorded and subsequently evaluated by a group of their peers as part of a study that examined how well they articulated their speech and how persuasively they presented their argument. Each participant was asked to deliver both speeches, and speech order was counterbalanced across participants.

The cold pressor test (CPT) - The CPT is one of the more widely used forms of experimental pain induction in psychological studies (see e.g., Russ et al., 1992). For this task, a cooler containing a $2^{\circ} \mathrm{C}$ (as indexed by a thermometer) mixture of crushed ice and water was placed on a stool next to the participant. A water circulator was placed in the cooler to prevent the water near the participant's hand from warming up. The participant was given instructions to place his/her hand (up to the wrist) into the water and to inform the researcher (a) when he/she first felt pain and (b) when the pain became intolerable. As soon as the participant's hand was submerged, the timer began. Participants were allowed to pull their hand out of the water whenever they pleased and were allowed to keep their hand in the water for a maximum of 2 min. Participants underwent the CPT twice, alternating the hand used for the CPT within the session, with hand order being counterbalanced across participants.

\section{Psychophysiological Measures}

Measures and settings were in accord with current methodological standards (Blumenthal et al., 2005). Eyeblink electromyography (EMG) responses were measured from the orbicularis oculi muscle with In Vivo Metric surface recording electrodes (Ag/AgCl, 11-mm outer diameter, 4-mm inner diameter contact surface) placed below the left eye. EMG activity of this muscle was amplified with a Biopac EMG amplifier and sampled $(1,000 \mathrm{~Hz})$ by a Biopac MP150 workstation that stored four versions of the EMG input: raw unfiltered EMG, filtered EMG in a passband of 28-500 Hz, a rectification of the filtered EMG signal, and a rectified and smoothed (five-sample boxcar filter) derivation of the filtered signal. The analyzed data were based on the smoothed EMG signal.

\section{Self-Report Measures}

Screening questionnaire-This questionnaire was designed to quickly measure NSSI and affect dysregulation levels. NSSI was screened with the item "In this past year, how often have you harmed or hurt your body on purpose (e.g., cutting or burning your skin, hitting yourself, etc.).” Affect dysregulation was screened with six items from the DERS (Gratz \& Roemer, 2004); these items had the highest factor loadings on each of the six factors of the DERS: Nonacceptance ("When I'm upset, I feel guilty for feeling that way"), Goals ("When I'm upset, I have difficulty concentrating"), Impulse ("When I'm upset, I lose control over my behaviors"), Awareness ("I am attentive to my feelings" [reverse-scored]), Strategies ("When I'm upset, I believe I'll end up feeling very depressed"), and Clarity ("I have difficulty making sense of my feelings"). All items are scored on a Likert-type scale ranging from 1 (almost never) to 5 (almost always).

Difficulties in Emotion Regulation Scale (DERS; Gratz \& Roemer, 2004)—The complete DERS is a 36-item questionnaire that shows high internal consistency, good test- 
retest reliability, and adequate construct and predictive validity; it is also positively correlated with NSSI in both men and women (Gratz \& Roemer, 2004).

Functional Assessment of Self-Mutilation (FASM; Lloyd-Richardson, 2008)The FASM is a 33-item measure that assesses type, frequency, and functions of NSSI (e.g., "[Have you ever] cut or carved your skin?" "[Did you do this] to feel relaxed?"). The FASM was utilized as a follow-up to the NSSI screening question in order to assure that the participants' NSSI behaviors fell into the "severe NSSI" category. Specifically, although the FASM asks about such behaviors as lip-biting and wound-picking, only participants endorsing severe NSSI behaviors such as skin cutting and burning were included in the NSSI group (see Table 1). Although hitting is sometimes a severe behavior, follow-up interviews suggested that no participants who indicated hitting as their most severe NSSI behavior engaged in severe hitting (i.e., causing tissue damage or bruising) or viewed their behavior as a form of self-injury (and did not endorse NSSI on the one-item NSSI screener). Accordingly, these participants $(n=3)$ were sorted into non-NSSI groups on the basis of their DERS scores; however, it should be noted that results were nearly identical when these participants were removed from analyses and when these participants were placed in the NSSI group.

Subjective Units of Distress Scale-Participants were asked to "rate your level of discomfort on a scale of 0 to 100 , with 0 indicating the most relaxed you have ever felt in your life, 50 being quite distressed but still functioning adequately, and 100 signifying the most distressed that you have ever felt in your life."

General health questionnaire-A general health questionnaire also was administered to monitor participants' use of psychoactive medications $(n=3)$ and tobacco $(n=6)$. Chisquare analyses indicated that neither medication nor tobacco use differed by group $(p s>$. 05 ), and univariate analyses showed that neither of these variables had a significant effect on psychophysiological variables $(p s>.05)$. Participants were also screened for current hearing-related illnesses (e.g., ruptured eardrums); however, no participants indicated any such illnesses.

\section{Data Analyses}

Startle-alone data-Blink response magnitude was calculated for each stimulus condition (see Blumenthal et al., 2005). Response magnitude was the average of the difference between peak and onset voltage of the smoothed EMG, within a window of 20-150 ms after stimulus onset for all trials; trials on which no response was found were assigned a value of 0 . Within a given block, the six startle-alone trials were averaged to determine the startlealone value for that block for each participant.

PPI data-PPI was calculated as the proportion of the difference from control (i.e., [prepulse condition - control condition]/control condition) for response magnitude, as recommended by Blumenthal, Elden, and Flaten (2004). Within a given block, the PPI trials were averaged to determine the PPI value for that block for each participant. 


\section{Data Analytic Plan}

Primary study hypotheses were examined using multiple-group (i.e., control, no-pain control, matched-AD, and NSSI) latent curve analyses. Specifically, a pre-post-post design using a structural equation modeling framework was utilized (Willoughby, Vandergrift, Blair, \& Granger, 2007). An important benefit of this design is the ability to examine changes in criterion measures over multiple experimental conditions while simultaneously controlling for possible differences in baseline (i.e., intercepts) across individuals (Willoughby et al., 2007). This approach also allows for a consideration of average levels of change within the sample as well as individual variability around this average. Three models - corresponding to the three sets of dependent variables (i.e., SUDS, startle-alone, and PPI) -were examined. All analyses were conducted using AMOS 17.0 (AMOS Development Corporation). Means and intercepts were estimated with full information direct maximum likelihood when data were missing.

The pre-post-post design involved the estimation of five latent factors from the five observed indicators (i.e., one indicator for each block of the experimental design). In each model, an initial latent construct (i.e., Baseline) represents initial levels of the dependent variable; paths were included between this latent construct and all five observed indicators. A second latent construct (i.e., Stress 1) is estimated from paths to the observed indicators at Block 2 after controlling for the path to Block 1. Thus, the estimated mean for the latent Stress 1 construct reflects changes in the dependent measure between Baseline and the exposure to the first stressor. A third latent construct (i.e., Pain 1) is estimated from a path to the third observed indicator, reflecting changes in the dependent measure between exposure to the first stressor and the first CPT. The fourth (Stress 2) and fifth (Pain 2) latent constructs follow a similar pattern: Stress 2 is estimated from paths to the observed indicator at Block 4, and Pain 2 is estimated from a path to the observed indicator at Block 5. Thus, Stress 2 represents changes in the dependent measure between Baseline and the second stressor, and Pain 2 represents changes in the dependent measure between the second stressor and the second CPT (see Figure 2). All paths are set to equal 1. For each observed indicator, intercepts are set to 0 ; error terms for each indicator have means and variances equal to 0 . Covariances are estimated between all five latent constructs.

For each of the three multigroup models (i.e., for each of the three sets of dependent variables), a baseline model was first estimated, allowing all latent parameters and covariances to vary freely across groups. This model was saturated but allowed for comparisons with subsequent models. Each of 10 individual covariance estimates was then fixed across groups, and individual chi-square difference tests were conducted to examine invariance across experimental groups (without significant detriment to model fit). A best fitting, parsimonious model was established once all covariances were set to be fixed, or to freely vary, on the basis of theoretical predictions and the results from these chi-square difference tests. Last, to examine primary hypotheses regarding group differences in stress reactivity and pain, a final set of model comparisons, with corresponding chi-square difference tests, was conducted to examine significant group differences in latent construct mean estimates. The presence of a significant chi-square difference test, suggesting decline in model fit after fixing a latent parameter across groups, suggested that the latent construct 
mean of one of the four experimental groups differed significantly from at least one of the others. Thus, this omnibus significance test was followed by contrast analyses.

\section{Results SUDS}

An initial multigroup pre-post-post structural equation model was conducted to examine subjective reports of distress during each block of the experiment. Participants' SUDS measures at Blocks 1-5 were used as observed indicators, and five latent factors were estimated, as described earlier (i.e., Baseline, Stress 1, Pain 1, Stress 2, Pain 2). Systematic testing of covariances across groups revealed that, compared with the saturated model (i.e., with all covariances and latent constructs freely varying across groups), seven covariances could be fixed across groups without significant detriment to model fit (see Table 2), $\Delta \chi ;^{2}(21)=23.74, n s$. Next, significant group differences in latent construct mean estimates were examined. Results revealed that the Baseline, Stress 1, and Stress 2 means could be fixed across groups without significant change in model fit, $\Delta \chi^{2}(9)=15.89, n s$. However, the means for Pain $1, \Delta \chi^{2}(3)=38.80, p<.0001$, and for Pain $2, \Delta \chi^{2}(3)=25.41, p<.0001$, were significantly moderated by group. Subsequent pairwise contrasts revealed that, as expected, the mean change in SUDS for the no pain group was significantly different from that for all other groups in Blocks 3 and 5, during which pain was administered to the other three groups. This indicated that whereas the control, matched-AD, and NSSI groups reported feeling more distressed after the CPT, the no pain group (which rested instead of undergoing the CPT) reported a decrease in distress. Of note, the NSSI group did not report a decrease in distress after the CPTs. The final model was an excellent fit to the data, $\chi^{2}(34)$ $=40.47, n s ; \chi^{2} / d f=1.19 ;$ comparative fit index $(\mathrm{CFI})=.99$; root-mean-square error of approximation $(\mathrm{RMSEA})=.04$.

Estimated latent construct means and all covariance estimates are listed in Table 2. As reflected in the estimated latent means, participants reported relatively low distress at Baseline, showed a significant increase in SUDS in the Stress 1 block, and evidenced another significant increase during the Pain 1 block (with the exception of the no pain group, which showed a significant decrease). A similar pattern was found for the Stress 2 and Pain 2 blocks (see Table 2, Figure 3). Overall, the SUDS results suggest that all groups (including the NSSI group) perceived the stress and pain manipulations as distressing.

\section{Startle-Alone}

A second multigroup pre-post-post structural equation model was conducted to examine startle responses during each block of the experiment. Analyses were conducted in a manner identical to those described earlier. Systematic testing of covariances across groups revealed that, compared with an initial saturated model (i.e., with all covariances and latent constructs freely varying across groups), eight covariances could be fixed across groups without significant detriment to model fit, $\Delta \chi^{2}(24)=35.31, n s$ (see Table 3). Next, significant group differences in latent construct mean estimates were examined. Results revealed that the Baseline, Stress 1, Stress 2, and Pain 2 means could be fixed across groups without significant change in model fit, $\Delta \chi^{2}(8)=10.42, n s$. Fixing the estimated mean for Pain 1 
across the four experimental groups did suggest a significant detriment in model fit, however, $\Delta \chi^{2}(3)=12.64, p<.01$. Thus, pairwise contrasts were conducted to examine significant differences in Pain 1 across groups. As with the results for SUDS, findings suggested that the no pain group was significantly different from all other groups. The final model was an excellent fit to the data, $\chi^{2}(42)=46.73, n s ; \chi^{2} / d f=1.23$; CFI $=.98$; RMSEA $=.05$.

Estimated latent construct means and all covariance estimates for the startle-alone results are listed in Table 3. As reflected in the estimated construct means, startle reactivity decreased for all four groups from Baseline to the Stress 1 block. From here, the startle reactivity of the no pain group remained constant, whereas the reactivity of the three groups that received the CPT continued to decrease (see Figure 4). Although this is consistent with the hypothesis that pain regulates affective valence in individuals with a history of NSSI, these results suggest that pain regulates affective valence for all groups. This unexpected finding is discussed in greater detail in the next section. Although startle reactivity increased during the Stress 2 block for the three groups that received the CPT, reactivity again decreased for these three groups in the Pain 2 block, replicating the Pain 1 effect.

\section{PPI}

A final multigroup pre-post-post structural equation model was conducted to examine PPI responses during each block of the experiment. These analyses were conducted in a manner identical to the analyses described earlier. Systematic testing of covariances across groups revealed that, compared with an initial saturated model (i.e., with all covariances and latent constructs freely varying across groups), seven covariances could be fixed across groups without significant detriment to model fit, $\Delta \chi^{2}(21)=26.10, n s$ (see Table 4). Next, significant group differences in latent construct mean estimates were examined. Results revealed that the Stress 1 and Stress 2 means could be fixed across groups without significant change in model fit, $\Delta \chi^{2}(9)=6.59, n s$. However, significant differences were revealed for Baseline, $\Delta \chi^{2}(3)=10.38, p<.05$, and Pain $1, \Delta \chi^{2}(3)=8.93, p<.05$.

Estimated latent construct means and all covariance estimates for PPI data are reported in Table 4. At Baseline, pairwise tests revealed that the control group displayed significantly higher PPI compared with the other groups. As reflected in the estimated latent construct means, PPI then decreased significantly for all four groups during the Stress 1 block. During the Pain 1 block, PPI continued to decrease significantly for the control and matched-AD groups. In contrast, PPI increased slightly (but non-significantly) for the no pain group and increased substantially and significantly for the NSSI group (see Figure 5). This NSSI pattern was significantly different from that for the control group, $\Delta \chi^{2}(1)=5.43, p<.05$, and the matched-AD group, $\Delta \chi^{2}(1)=4.20, p<.05$. This finding is highly consistent with hypotheses, suggesting that whereas other groups experienced cognitive dysregulation after the CPT, the NSSI group experienced cognitive regulation after the CPT. Similar mean PPI patterns were observed for the Stress 2 and Pain 2 blocks (see Table 4 and Figure 4). This final model was an excellent fit to the data, $\chi^{2}(33)=33.61, n s ; \chi^{2} / d f=1.02$; CFI $=.99$; RMSEA $=.01$. Overall, these data provide strong evidence in support of a cognitive regulation function for NSSI. 
Although this model does not indicate group differences during the Pain 2 block (see Table 4), this is likely because our statistical model essentially controls for the Pain 1 effect when testing differences at Pain 2. In examining the Pain 2 differences independent of the Pain 1 effect, analyses revealed that there were no significant differences between the NSSI and matched-AD groups, $\Delta \chi^{2}(1)=1.05, n s$. In contrast, the control group remained significantly different from the NSSI group during the Pain 2 block, $\Delta \chi^{2}(1)=3.88, p<.05$. Thus, the Pain 2 results provide a partial replication of the Pain 1 results but suggest that the matched$\mathrm{AD}$ group's responses to pain may have been altered as a result of earlier experimental manipulations.

\section{Discussion}

The present study was designed to address several gaps in the understanding of the functions of NSSI. On a methodological level, findings suggested that the results of laboratory NSSI studies may be generalized to nonclinical samples and that affect dysregulation does not completely explain the abnormal responses to pain of individuals with a history of NSSI. Additionally, although inducing acute negative affect before the NSSI-proxy may provide increased ecological validity, results indicated this leads to findings similar to those obtained in other laboratory NSSI studies when negative affect is not induced. On a more conceptual level, the present study provided the first psychophysiological support for the hypotheses that NSSI serves to regulate cognitive processing and affective valence. Moreover, the unexpected finding of a reduction in startle reactivity in the control groups after the NSSIproxies may provide an important window into the development of NSSI. However, as discussed later in the Discrepancies With Self-Report Data section, self-report results were inconsistent with psychophysiological findings.

\section{Support for a Cognitive Regulation Function}

The PPI results supported the hypothesis that the NSSI and matched-AD groups display poorer cognitive processing at baseline. This is consistent with other studies that have shown that PPI is lower at baseline in individuals diagnosed with internalizing disorders (see Franklin et al., 2009), externalizing disorders (see e.g., Kumari et al., 2005), and impulsecontrol disorders (see e.g., Stojanov et al., 2003). This finding indicates that NSSI is associated with decreased activity in the areas of the brain that undergird executive functioning (Bitsios et al., 2006; Inglis, Olmstead, \& Robbins, 2001; Winn, 2006) and increased activity in the areas of the brain associated with affective reactivity (Swerdlow et al., 2001; Winn, 2006). Such a constellation of neurological activity is consistent with the neurological concomitants of affect dysregulation (Brown, Manuck, Flory, \& Hariri, 2006; Ochsner \& Gross, 2008; Schmahl et al., 2006) and should constitute a system that is biased toward engaging in impulsive affect-focused behaviors such as NSSI.

The PPI results also provided the first support for the hypothesis that, in addition to serving an affect regulation function, NSSI also serves a cognitive regulation function. The PPI results across the four postbaseline blocks supported this hypothesis: After both NSSIproxies, PPI significantly increased — even beyond baseline levels—for the NSSI group, whereas it decreased in these conditions for the control group (see Figure 5). The results of 
the matched-AD group were less clear, because their PPI improved slightly after the second NSSI-proxy. As discussed later, this may suggest that individuals with higher affect dysregulation are more vulnerable to the reinforcing properties of the offset of pain. Nevertheless, the levels of affect dysregulation in the present study were low and restricted; accordingly, the present results may not be able to speak to "dysregulation." The PPI results of the no pain group increase confidence that these effects were due to the NSSI-proxy. This is because, without the NSSI-proxy, PPI improves slightly for the no pain group rather than significantly increasing as in the NSSI group or significantly decreasing as in the control group. Moreover, confidence is increased in this finding because the PPI results of the first stressor/NSSI-proxy blocks are nearly identical to the results of the second stressor/NSSIproxy blocks. The NSSI group's increase in PPI after the NSSI-proxies is consistent with the pattern of brain activity observed in the self-injury group of Schmahl et al. (2006), which is consistent with the neural correlates of both improved executive functioning and increased PPI.

Although only a handful of PPI studies have employed experimental designs or even examined PPI beyond baseline, there is some precedence for these findings. Duley et al. (2007) measured PPI in undergraduates high and low in trait anxiety and found that, whereas the high-anxiety group displayed decreased PPI at baseline, this effect disappeared after each group underwent 30 min of exercise. Similar to the results of Duley et al. concerning exercise and anxiety, the present results suggest that for some individuals NSSI is an effective way to regulate cognitive processing. Indeed, this parallel is consistent with Wallenstein and Nock (2007), who showed that exercise may be an adaptive substitute for NSSI. However, in the present study it is notable that the NSSI group's highest PPI levels are roughly equivalent to the control group's lowest PPI levels (both occurring after the NSSI-proxy). This suggests that even post-NSSI, individuals with a history of NSSI may have poor cognitive processing.

\section{Evidence for Affective Valence Regulation}

The NSSI group displayed, consistent with an affective valence regulation function of NSSI, decreased startle-alone reactivity after the NSSI-proxy. However, contrary to expectations, the control and matched-AD groups showed this same pattern (see Figure 4). Moreover, the first few blocks appear to be contaminated with startle habituation as reactivity steadily decreased across these blocks. Indeed, there are no significant between-groups differences in startle-alone reactivity between the NSSI, matched-AD, and control groups. Ruling out habituation as an explanation for this finding, the results of the no pain group demonstrated that, without the NSSI-proxy, reactivity levels off after the first stress block. This supports the interpretation that the decreased reactivity observed in the other two groups after the NSSI-proxy was due to a shift away from negative affective valence. For the NSSI group, this finding is highly consistent with the ANR function and expectations; nonetheless, for the control and matched-AD groups, this finding seems counterintuitive.

Further examination of the literature, however, reveals that this finding is consistent with previous pain studies and the opponent process theory of acquired motivation (Joiner, 2005; Solomon, 1980). Previous human studies have demonstrated that startle-alone reactivity is 
decreased by the offset of pain and cues associated with the offset of pain (Andreatta, Muhlberger, Yarali, Gerber, \& Pauli, 2010; Tavernor, Abduljawad, Langley, Bradshaw, \& Szabadi, 2000). Similarly, Walker, Cassella, Lee, De Lima, and Davis (1997) found that, in rats, startle-alone reactivity increased as the intensity of a painful shock increased up to a certain point, at which time startle-alone reactivity became inversely related to shock intensity. Because participants in the present study remained in the CPT until it became too painful to tolerate, the present results might be explained by this same stress-induced analgesia effect. Additionally, because the present study measured variables in the minutes after the CPT, it is important to note that the present study may have measured responses to the relief from pain rather than direct responses to pain. In terms of opponent processes (Solomon, 1980), the CPT itself induces negative affect for everyone (i.e., the primary process), whereas termination of the CPT causes a shift toward the opposite affective valence for everyone (i.e., the opponent process). According to this theory (Solomon, 1980), after repeated experience with the stimulus, the primary process decreases in intensity, whereas the opponent process becomes more intense. Joiner (2005) suggested that this may help to explain why self-injurers have increased pain tolerance (decreased primary process) and find pain reinforcing (increased opponent process).

Consistent with the application of opponent process theory to drug research (Koob, 2009), it may be that NSSI (or its offset) is initially positively reinforcing and later becomes negatively reinforcing. This hypothesis is supported when the affective valence results of the present study are combined with the affective arousal results of previous laboratory NSSI studies. In sum, these studies have shown that NSSI groups display decreased arousal and a shift away from negative valence, indicating a reduction in negative affect (see Lang et al., 1990). This supports an ANR function for individuals with NSSI experience. In contrast, control groups have shown increased arousal and a shift away from negative valence, suggesting increased positive affect (see Lang et al., 1990). This supports an APR function for individuals with no NSSI experience. This difference may also help to explain why both the ANR and APR functions are commonly endorsed in NSSI research (Klonsky, 2007). Inasmuch as PPI varies with arousal (Grillon \& Davis, 1997), this hypothesis is also supported by the present results because, postproxy, the NSSI group showed increased PPI (decreased arousal) and the control group evidenced decreased PPI (increased arousal). Indeed, this shift from APR to ANR may have been observed in the present study because the matched-AD group displayed decreased PPI after the first NSSI-proxy (suggesting APR) and slightly increased PPI after the second NSSI-proxy (suggesting ANR). This possible shift should be more likely to occur in individuals higher in affect dysregulation because increased reactivity to a stimulus should activate its opponent process more intensely (Solomon, 1980). Nevertheless, this intriguing APR-to-ANR hypothesis has not been directly investigated and remains speculative; future studies should explore this possibility.

We were surprised that the non-NSSI groups displayed affective valence regulation after the CPTs because it is often emphasized that repeated stimulation increases the intensity of opponent processes (see e.g., Joiner, 2005). However, as described by Solomon (1980), intense primary processes (e.g., intolerable pain) can also instantiate intense opponent processes (e.g., intense relief) on the first trial of learning. This effect may partially explain the non-NSSI startle-alone results. Nevertheless, future studies should examine factors other 
than opponent processes that may contribute to the reinforcement of NSSI. Specifically, cognitive processes (e.g., placebo analgesia), social influence (e.g., socially induced placebo analgesia), and genetics may influence NSSI reinforcement.

\section{Discrepancies With Self-Report Data}

Although the psychophysiological data were consistent with hypotheses, there were no significant SUDS differences between the NSSI, matched-AD, and control groups after the NSSI-proxies (see Figure 3). This discrepancy is similar to the results of Andreatta et al. (2010), who found that participants evaluated all pain-relevant cues as "emotionally negative," whereas their startle reactivity was reduced in the presence of cues associated with the offset of pain. In accordance with Janis and Nock (2009), the present results suggest that individuals with a history of NSSI may not be consciously aware of the processes underlying NSSI, and accordingly, self-report measures may be a less reliable index of these processes. However, this remains speculative.

Of note, this finding continues a trend of somewhat inconsistent self-report findings in laboratory NSSI studies. Although each of these studies has consistently found evidence of reduced physiological arousal after an NSSI-proxy, their self-report data have not been consistently correlated with their physiological data. One of these studies did not include affect-related self-report data (Schmahl et al., 2006) and another did not include physiological measures (Russ et al., 1992). The three others found reductions on selfreported arousal measures during the "incident" phase of their NSSI scripts, but they generally did not find reductions in other affective measures during this phase, and the selfreported arousal reductions were not always specific to the NSSI script (Brain et al., 1998; Haines et al., 1995; Welch et al., 2008). These inconsistencies echo the NSSI-impulsivity findings of Janis and Nock (2009), general findings from social and cognitive psychology (see e.g., Nisbett \& Wilson, 1977), and findings from other startle-based studies (see e.g., Patrick et al., 1993). Such inconsistencies do not invalidate either self-report or psychophysiological methods; rather, they suggest that future studies should continue to employ multiple methods of measurement and investigate the reasons for these discrepancies.

\section{Limitations and Future Directions}

The present study should be interpreted in accord with its limitations. First, the sample of the present study included only college students, which may limit the generalization of the present findings to more clinically severe samples. Nonetheless, the present findings are consistent with those of previous clinical studies, suggesting that NSSI functions are similar across clinical and nonclinical samples and that extremely high frequency rates of NSSI are not necessary to observe cognitive-affective regulation after an NSSI-proxy. Future studies, however, would benefit from replicating the present affective valence and cognitive regulation findings in a more clinically severe and representative sample. Second, although the present results support the hypothesis that affect dysregulation does not play a large role in the abnormal pain processing associated with NSSI, the range and degree of affect dysregulation in the present study were restricted. Replicating this finding in a clinical sample would provide much stronger support for this hypothesis. Third, the present NSSI 
sample $(n=16)$ was small, and generalizations beyond this sample should accordingly be tempered. Of note, however, this represents the largest nonscript-based NSSI-proxy study to date ( $n=11$ for Russ et al., 1992; $n=12$ for Schmahl et al., 2006). Nonetheless, future painbased laboratory NSSI studies certainly should employ larger samples. Fourth, the present study and other laboratory studies assume that the NSSI-proxy causes the physiological differences observed in the NSSI groups. Although this interpretation is bolstered by no pain group results in the present study, it is possible that top-down cognitive strategies also play a role in this regulation (cf. Dillon \& LaBar, 2005). Future studies should investigate this possibility.

Fifth, it is not clear how well the stress induction and NSSI-proxy approximate the actual NSSI process. In examining the laboratory NSSI literature as a whole, however, it appears that different types of NSSI-proxies (e.g., heat pain, cold pain, imaginary scripts) yield similar results. This suggests that the results of the present study are at least as ecologically valid as those of other studies. Although one major limitation of laboratory NSSI studies is that they lack ecological validity due to their use of a proxy, preliminary results from a naturalistic psychophysiological study of NSSI are highly consistent with the results of these studies (Nock, Mendes, Deliberto, \& Dour, 2009).

Sixth, although startle modulation measures are well-validated and widely used psychophysiological tools for the investigation of affect and information processing, they have their limitations. For all startle modulation measures, the primary limitation is that startle stimuli must be presented throughout the experiment; this could conceivably affect ongoing processes relevant to cognitive-affective regulation. This limitation is shared by many performance-based and psychophysiological measures (e.g., event-related potentials). Providing some confidence for the use of such measures in laboratory NSSI studies, the results of the present study are consistent with those of studies that employed more passive measures (e.g., autonomic measures, fMRI). Additionally, although startle reactivity can discriminate between pleasant and neutral affects, it is most effective as a measure of negative affect (Vaidyanathan et al., 2009). Measures such as the postauricular reflex would be more effective at measuring positive affect (Benning, Patrick, \& Lang, 2004). Finally, although PPI is a well-validated measure of information processing, other measures of information processing (e.g., P50, P300) should be employed in future studies to provide complementary information about cognitive regulation in NSSI.

\section{Conclusions}

The present results supported the hypotheses that, in addition to serving an arousal reduction function, NSSI also serves to reduce negative affective valence and to improve cognitive processing. In addition, the present study supported the hypotheses that NSSI serves the same function in clinical and nonclinical populations and that affect dysregulation differences do not explain the association between NSSI and abnormal pain processing. The unexpected finding of affective valence regulation postproxy in the control group is consistent with the hypothesis that opponent processes play a role in self-injury (Joiner, 2005). This suggests that NSSI initially serves an APR function and later serves an ANR function (cf. Koob, 2009); nevertheless, this hypothesis remains largely untested. Future 
studies should build on the present findings and continue to provide much-needed information about why people engage in NSSI.

\section{Acknowledgments}

This study was supported in part by a National Science Foundation Graduate Research Fellowship and a student research grant from the International Society for Traumatic Stress Studies (both awarded to Joseph C. Franklin) and a Summer Undergraduate Research Fellowship from the University of North Carolina awarded to Elenda T. Hessel. The authors wish to thank Gabriel S. Dichter, Jonathan S. Abramowitz, John D. Guerry, and Terry D. Blumenthal for their comments on a draft of this article. The authors would also like to thank Megan L. Brandon, Eleanor K. Hanna, and Michael G. Wheaton for their invaluable assistance with data collection.

\section{References}

Andreatta M, Muhlberger A, Yarali A, Gerber B, Pauli P. A rift between implicit and explicit condition valence in human pain relief learning. Proceedings of the Royal Society B. 2010; 277:2411-2416. [PubMed: 20356893]

Benning SD, Patrick CJ, Lang AR. Emotional modulation of the post-auricular reflex. Psychophysiology. 2004; 41:426-432.10.1111/j.1469-8986.00160.x [PubMed: 15102128]

Bitsios P, Giakoumaki SG. Relationship of prepulse inhibition of the startle reflex to attentional and executive mechanisms in man. International Journal of Psychophysiology. 2005; 55:229241.10.1016/j.ijpsycho.2004.08.002 [PubMed: 15649554]

Bitsios P, Giakoumaki S, Theou K, Frangou S. Increased prepulse inhibition of the acoustic startle response is associated with better strategy formation and execution times in healthy males. Neuropsychologia. 2006; 44:2494-2499.10.1016/j.neuropsychologia.2006.04.001 [PubMed: 16698050]

Blumenthal, TD. Short lead interval modification. In: Dawson, ME.; Schell, AM.; Bohmelt, AH., editors. Startle modification: Implications for neuroscience, cognitive science, and clinical science. Cambridge, England: Cambridge University Press; 1999. p. 51-71.

Blumenthal TD, Cuthbert BN, Filion DL, Hackley S, Lipp OV, van Boxtel A. Committee report: Guidelines for human startle eyeblink electromyographic studies. Psychophysiology. 2005; 42:115.10.1111/j.1469-8986.2005.00271.x [PubMed: 15720576]

Blumenthal TD, Elden A, Flaten MA. A comparison of several methods used to quantify prepulse inhibition of eyeblink responding. Psychophysiology. 2004; 41:326-332.10.1111/j. 1469-8986.2003.00144.x [PubMed: 15032998]

Blumenthal TD, Noto JV, Fox MA, Franklin JC. Background noise decreases both prepulse elicitation and inhibition of acoustic startle blink responding. Biological Psychology. 2006; 72:173179.10.1016/j.biopsycho.2005.10.001 [PubMed: 16303226]

Bradley, MM.; Cuthbert, BN.; Lang, PJ. Affect and the startle reflex. In: Dawson, ME.; Schell, AM.; Bohmelt, AH., editors. Startle modification: Implications for neuroscience, cognitive science, and clinical science. Cambridge, England: Cambridge University Press; 1999. p. 157-183.

Braff D, Stone C, Callaway E, Geyer M, Glick I, Bali L. Prestimulus effects on human startle reflex in normals and schizophrenics. Psychophysiology. 1978; 15:339-343.10.1111/j. 1469-8986.1978.tb01390.x [PubMed: 693742]

Brain KL, Haines J, Williams CL. The psychophysiology of self-mutilation: Evidence of tension reduction. Archives of Suicide Research. 1998; 4:227-242.10.1080/13811119808258298

Brown SM, Manuck SB, Flory JD, Hariri AR. Neural basis of individual differences in impulsivity: Contributions of corticolimbic circuits for behavioral arousal and control. Emotion. 2006; 6:239245.10.1037/1528-3542.6.2.239 [PubMed: 16768556]

Cadenhead KS, Geyer MA, Braff DL. Impaired startle prepulse inhibition and habituation in patients with schizotypal personality disorder. American Journal of Psychiatry. 1993; 150:1862-1867. [PubMed: 8238643]

Darche MA. Psychological factors differentiating self-mutilating and non-self mutilating adolescent inpatient females. Psychiatric Hospital. 1990; 21:31-35. 
Dawson ME, Schell AM, Hazlett EA, Nuechterlein KH, Filion DL. On the clinical and cognitive meaning of impaired sensorimotor gating in schizophrenia. Psychiatry Research. 2000; 96:187197.10.1016/S0165-1781(00)00208-0 [PubMed: 11084215]

DiClemente RJ, Ponton LE, Hartley D. Prevalence and correlates of cutting behavior: Risk for HIV transmission. Journal of the American Academy of Child and Adolescent Psychiatry. 1991; 30:735-739.10.1097/00004583-199109000-00006 [PubMed: 1938787]

Dillon DG, LaBar KS. Startle modulation during conscious emotion regulation is arousal-dependent. Behavioral Neuroscience. 2005; 119:1118-1124.10.1037/0735-7044.119.4.1118 [PubMed: 16187839]

Duley AR, Hillman CH, Coombes S, Janelle CM. Sensorimotor gating and anxiety: Prepulse inhibition following acute exercise. International Journal of Psychophysiology. 2007; 64:157-164.10.1016/ j.ijpsycho.2007.01.006 [PubMed: 17350126]

Duncan S, Barrett LF. Affect as a form of cognition: A neurobiological analysis. Cognition \& Emotion. 2007; 21:1184-1211.10.1080/02699930701437931 [PubMed: 18509504]

Franklin JC, Bowker KB, Blumenthal TD. The association between anxiety and prepulse inhibition of acoustic startle in a normative sample: The importance of signal-to-noise ratio. Personality and Individual Differences. 2009; 46:369-373.10.1016/j.paid.2008.11.004

Gratz KL, Roemer L. Multidimensional assessment of emotion regulation and dysregulation: Development, factor structure, and initial validation of the Difficulties in Emotion Regulation Scale. Journal of Psychopathology and Behavioral Assessment. 2004; 26:41-54.10.1023/B:JOBA. 0000007455.08539 .94

Gratz KL, Roemer L. The relationship between emotion dysregulation and deliberate self-harm among female undergraduate students at an urban commuter university. Cognitive Behaviour Therapy. 2008; 37:14-25.10.1080/16506070701819524 [PubMed: 18365795]

Grillon C, Davis M. Effects of stress and shock anticipation on prepulse inhibition of the startle reflex. Psychophysiology. 1997; 34:511-517.10.1111/j.1469-8986.1997.tb01737.x [PubMed: 9299905]

Haines J, Williams CL, Brain KL, Wilson GV. The psychophysiology of self-mutilation. Journal of Abnormal Psychology. 1995; 104:471-489.10.1037/0021-843X.104.3.471 [PubMed: 7673571]

Inglis WL, Olmstead MC, Robbins TW. Selective deficits in attentional performance on the 5-choice serial reaction time task following pedunculopontine tegmental nucleus lesions. Behavioural Brain Research. 2001; 123:117-131.10.1016/S0166-4328(01)00181-4 [PubMed: 11399325]

Janis IB, Nock MK. Are self-injurers impulsive? Results from two behavioral laboratory studies. Psychiatry Research. 2009; 169:261-267.10.1016/j.psychres.2008.06.041 [PubMed: 19758706]

Joiner, T. Why people die by suicide. Cambridge, MA: Harvard University Press; 2005.

Klonsky ED. The functions of deliberate self-injury: A review of the evidence. Clinical Psychology Review. 2007; 27:226-239.10.1016/j.cpr.2006.08.002 [PubMed: 17014942]

Klonsky ED. The functions of self-injury in young adults who cut themselves: Clarifying the evidence for affect-regulation. Psychiatry Research. 2009; 166:260-268.10.1016/j.psychres.2008.02.008 [PubMed: 19275962]

Klossika I, Flor H, Kamping S, Bleichhardt G, Trautmann N, Treede R, Schmahl C. Emotional modulation of pain: A clinical perspective. Pain. 2006; 124:264-268.10.1016/j.pain.2006.08.007 [PubMed: 16934927]

Koch M, Fendt M. Startle response modulation as a behavioral tool in neuropharmacology. Current Neuropharmacology. 2003; 1:175-185.10.2174/1570159033477161

Koob GF. Dynamics of neuronal circuits in addiction: Reward, antireward, and emotional memory. Pharmacopsychiatry. 2009; 42(Suppl 1):S32-S41.10.1055/s-0029-1216356 [PubMed: 19434554]

Kumari V, Das M, Hodgins S, Zachariah E, Barkataki I, Howlett M, Sharma T. Associations between violent behaviour and impaired prepulse inhibition of the startle response in antisocial personality disorder and schizophrenia. Behavioural Brain Research. 2005; 158:159-166.10.1016/j.bbr. 2004.08.021 [PubMed: 15680203]

Lang PJ, Bradley MM, Cuthbert BN. Emotion, attention, and the startle reflex. Psychological Review. 1990; 97:377-395.10.1037/0033-295X.97.3.377 [PubMed: 2200076]

Linehan, MM. Cognitive-behavior treatment of borderline personality disorder. New York, NY: Guilford Press; 1993. 
Lloyd-Richardson E. Adolescent nonsuicidal self-injury: Who is doing it and why? Journal of Developmental and Behavioral Pediatrics. 2008; 29:216-218.10.1097/DBP.0b013e318173a5c1 [PubMed: 18550991]

Nisbett RE, Wilson TD. Telling more than we can know: Verbal reports on mental processes. Psychological Review. 1977; 84:231-259.10.1037/0033-295X.84.3.231

Nock MK, Joiner TE, Gordon KH, Lloyd-Richardson E, Prinstein MJ. Non-suicidal self-injury among adolescents: Diagnostic correlates and relation to suicide attempts. Psychiatry Research. 2006; 144:65-72.10.1016/j.psychres.2006.05.010 [PubMed: 16887199]

Nock, MK.; Mendes, WB.; Deliberto, TL.; Dour, HJ. Evidence for the affect regulating properties of self-injury: Results from a real-time physiological monitoring study. In: Hooley, JM.; Nock, MK., Co-chairs, editors. New conceptual and methodological approaches to the study of self-injury; Symposium conducted at the 43rd Annual Convention of the Association for Behavioral and Cognitive Therapies; New York, NY.. 2009 Nov.

Nock MK, Prinstein MJ. A functional approach to the assessment of self-mutilative behavior. Journal of Consulting and Clinical Psychology. 2004; 72:885-890.10.1037/0022-006X.72.5.885 [PubMed: 15482046]

Ochsner K, Gross J. Cognitive emotion regulation: Insights from social cognitive and affective neuroscience. Current Directions in Psychological Science. 2008; 17:153-158.10.1111/j. 1467-8721.2008.00566.x

Patrick CJ, Bradley MM, Lang PJ. Emotion in the criminal psychopath: Startle reflex modulation. Journal of Abnormal Psychology. 1993; 102:82-92.10.1037/0021-843X.102.1.82 [PubMed: 8436703]

Russ MJ, Roth SD, Lerman A, Kakuma T, Harrison K, Shindle-decker RD, Mattis S. Pain perception in self-injurious patients with borderline personality disorder. Biological Psychiatry. 1992; 32:501-511.10.1016/0006-3223(92)90218-O [PubMed: 1445967]

Schmahl C, Bohus M, Esposito F, Treede R, Di Salle F, Greffrath W, Seifritz E. Neural correlates of antinociception in borderline personality disorder. Archives of General Psychiatry. 2006; 63:659667.10.1001/archpsyc.63.6.659 [PubMed: 16754839]

Solomon RL. The opponent-process theory of acquired motivation: The costs of pleasure and the benefits of pain. American Psychologist. 1980; 35:691-712.10.1037/0003-066X.35.8.691 [PubMed: 7416563]

Stojanov W, Karayanidis F, Johnston P, Bailey A, Carr V, Schall U. Disrupted sensory gating in pathological gambling. Biological Psychiatry. 2003; 54:474-484.10.1016/S0006-3223(02)01745-6 [PubMed: 12915292]

Swerdlow NR, Geyer MA, Braff DL. Neural regulation of prepulse inhibition of startle in the rat: Current knowledge and future challenges. Psychopharmacology. 2001; 156:194-215.10.1007/ s002130100799 [PubMed: 11549223]

Tavernor SJ, Abduljawad KAJ, Langley RW, Bradshaw CM, Szabadi E. Effects of pentagastrin and the cold pressor test on the acoustic startle response and pupillary function in man. Journal of Psychopharmacology. 2000; 14:387-394.10.1177/026988110001400407 [PubMed: 11198057]

Vaidyanathan U, Patrick C, Cuthbert B. Linking dimensional models of internalizing psychopathology to neurobiological systems: Affect-modulated startle as an indicator of fear and distress disorders and affiliated traits. Psychological Bulletin. 2009; 135:909-942.10.1037/a0017222 [PubMed: 19883142]

Walker DL, Cassella JV, Lee Y, De Lima TCM, Davis M. Opposing roles of the amygdala and dorsolateral periaqueductal gray in fear-potentiated startle. Neuroscience \& Biobehavioral Review. 1997; 21:743-753.10.1016/S0149-7634(96)00061-9

Wallenstein MB, Nock MK. Physical exercise as a treatment for non-suicidal self-injury: Evidence from a single case study. American Journal of Psychiatry. 2007; 164:350-351.10.1176/appi.ajp. 164.2.350-a [PubMed: 17267807]

Welch SS, Linehan MM, Sylvers P, Chittams J, Rizvi SL. Emotional responses to self-injury imagery among adults with borderline personality disorder. Journal of Consulting and Clinical Psychology. 2008; 76:45-51.10.1037/0022-006X.76.1.45 [PubMed: 18229982] 
Whitlock J, Eckenrode J, Silverman D. Self-injurious behaviors in a college population. Pediatrics. 2006; 117:1939-1948.10.1542/peds.2005-2543 [PubMed: 16740834]

Willoughby M, Vandergrift N, Blair C, Granger DA. A structural equation modeling approach for the analysis of cortisol data using pre-post-post designs. Structural Equation Modeling. 2007; 14:125145.10.1207/s15328007sem1401_7

Winn P. How best to consider the structure and function of the pedunculopontine tegmental nucleus: Evidence from animal studies. Journal of the Neurological Sciences. 2006; 248:234-250. [PubMed: 16765383]

Yee CM, Mathis KI, Sun JC, Sholty GL, Lang PJ, Bachman P, Nuechterlein KH. Integrity of emotional and motivational states during the prodromal, first-episode, and chronic phases of schizophrenia. Journal of Abnormal Psychology. 2010; 119:71-82.10.1037/a0018475 [PubMed: 20141244] 


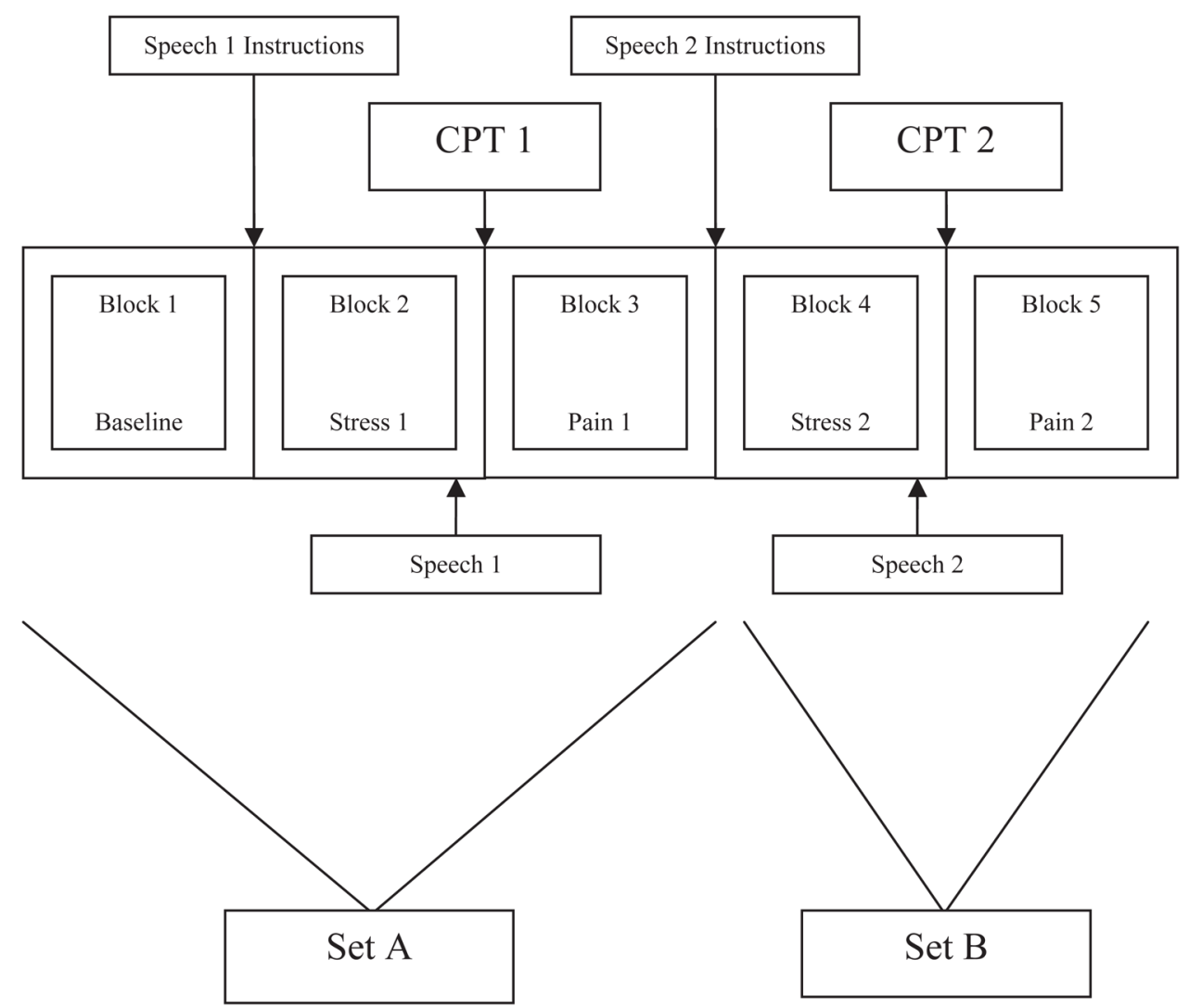

Figure 1.

Diagram of the experimental design. There were intervals of 3 min between each block of stimuli (intervals were filled with rest or instructions/speech or the CPT). CPT $=$ cold pressor test. 


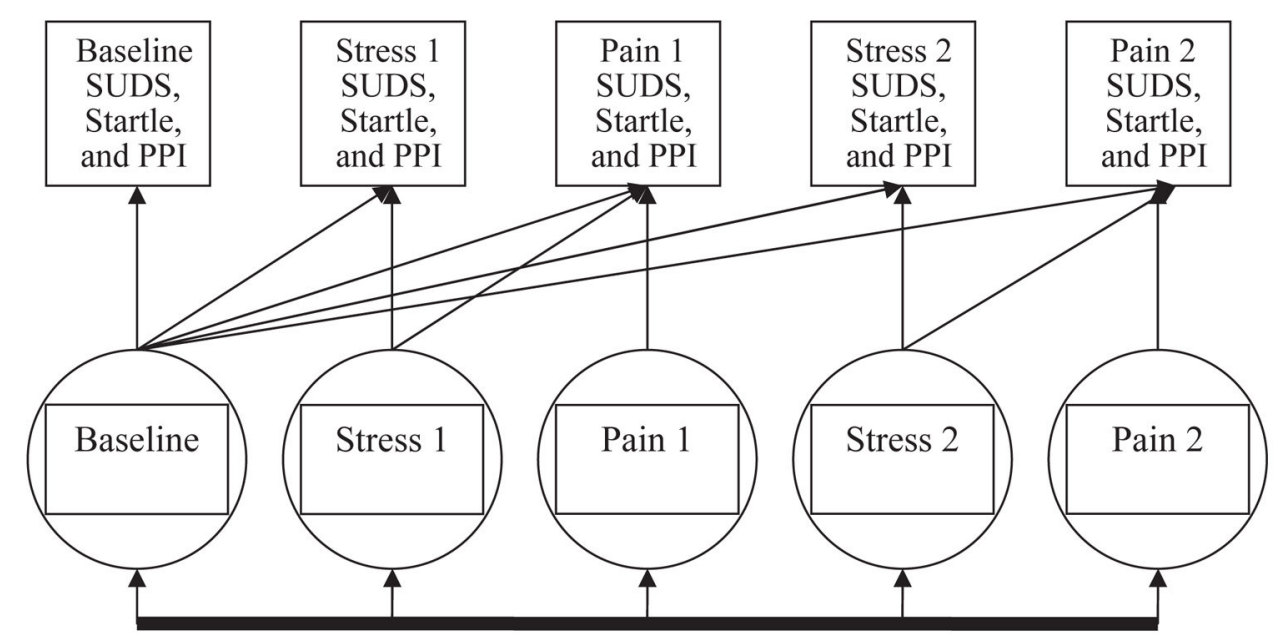

Figure 2.

Pre-post-post structural equation modeling diagram. SUDS = subjective units of distress; PPI $=$ prepulse inhibition. 


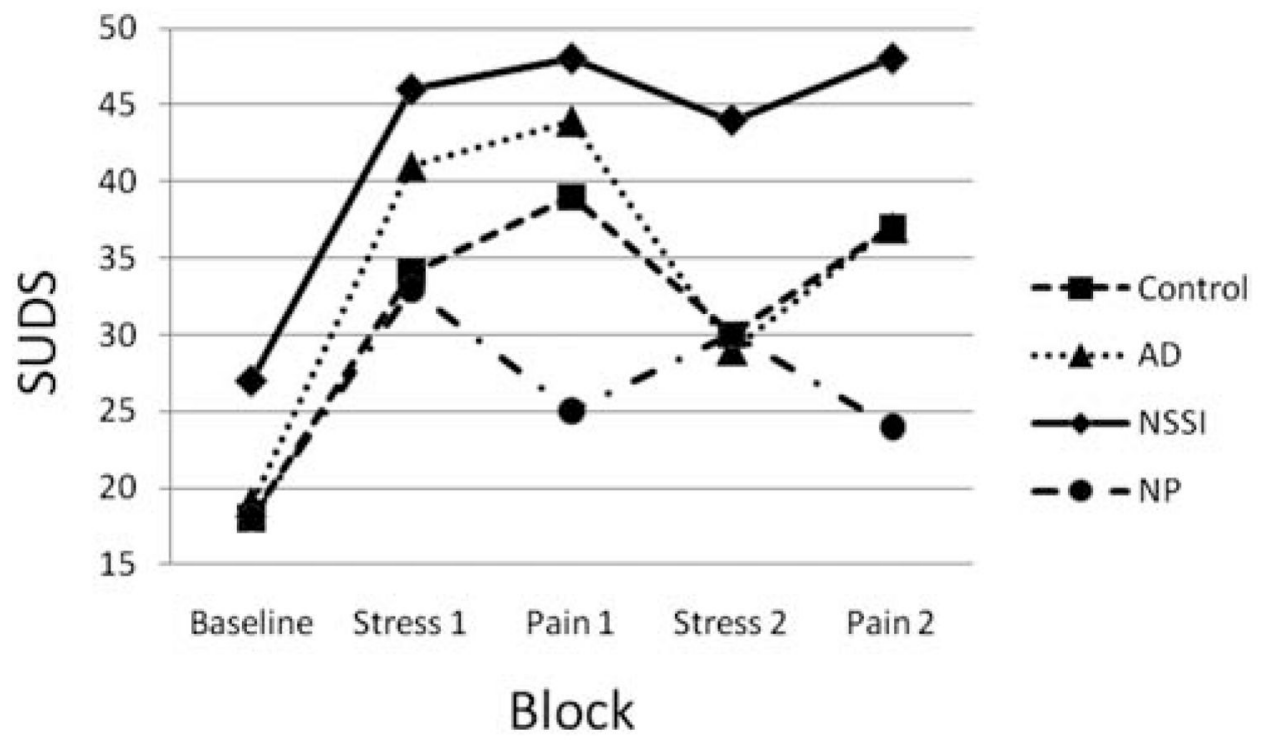

Figure 3.

Mean subjective units of distress (SUDS) across blocks by group. AD = matched affect dysregulation group; NSSI = nonsuicidal self-injury group; NP = no pain group. 


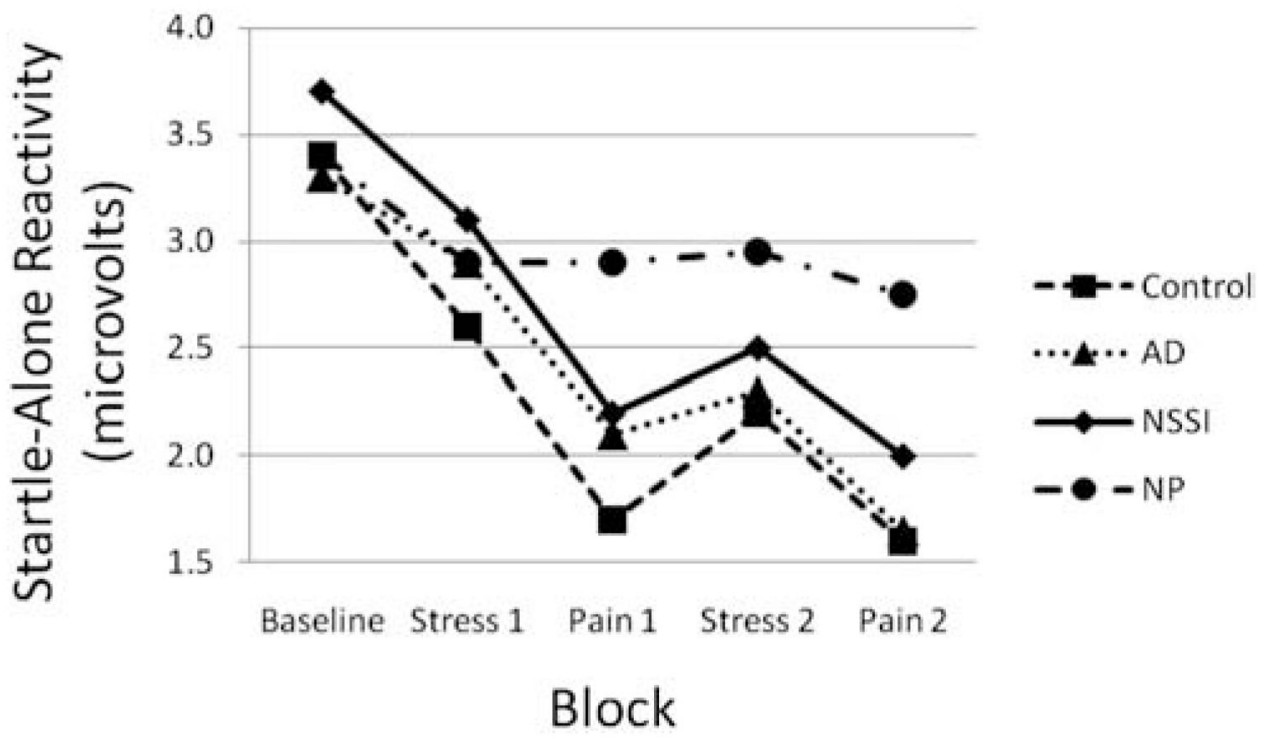

Figure 4.

Mean startle-alone reactivity across blocks by group. $\mathrm{AD}=$ matched affect dysregulation group; $\mathrm{NSSI}=$ nonsuicidal self-injury group; $\mathrm{NP}=$ no pain group . 


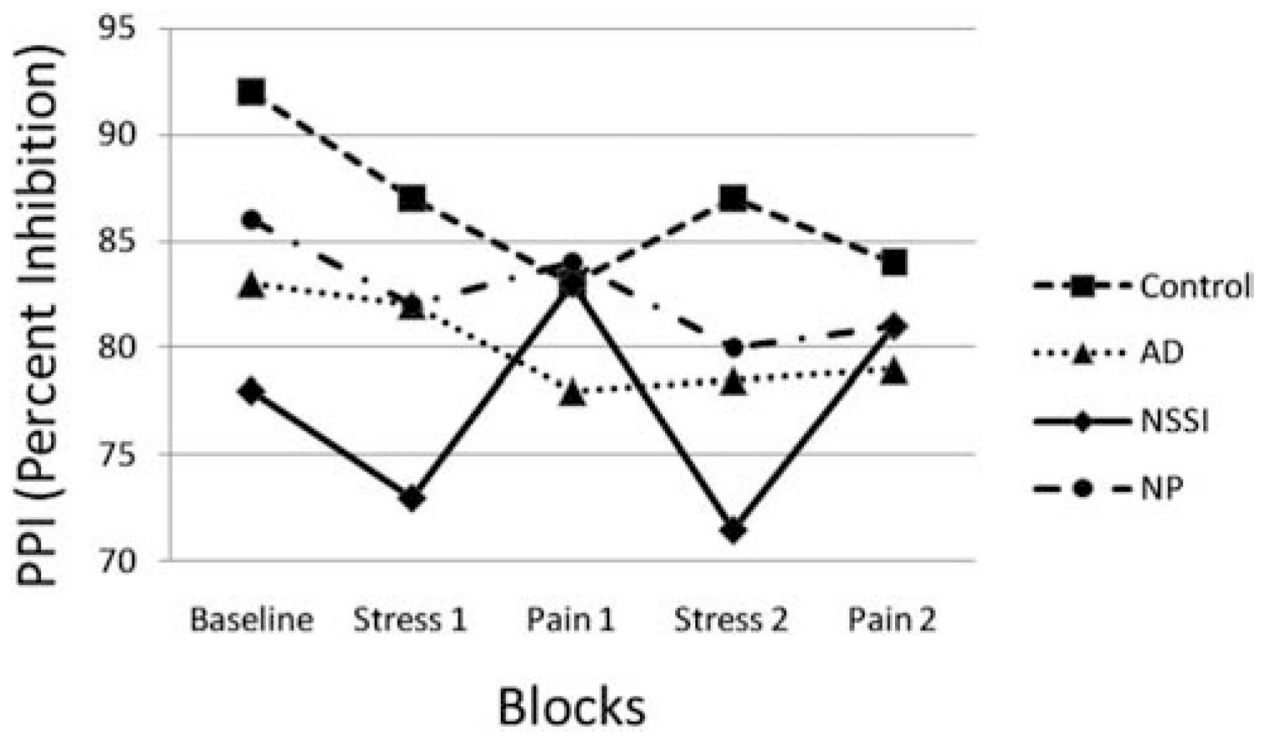

Figure 5.

Mean prepulse inhibition (PPI) across blocks by group. $\mathrm{AD}=$ matched affect dysregulation group; NSSI = nonsuicidal self-injury group; $\mathrm{NP}=$ no pain group . 


\section{Table 1}

Self-Injury Data of the NSSI Group (Past Year)

\begin{tabular}{llll}
\hline & & \multicolumn{2}{c}{ Times injured } \\
\cline { 3 - 4 } Injury type & Endorsing $(\%)$ & $\boldsymbol{M}(\boldsymbol{S D})$ & Range $^{\boldsymbol{a}}$ \\
\hline Cut & 62.5 & $5.86(5.50)$ & $2-15$ \\
Hit & 37.5 & $6.43(5.44)$ & $1-15$ \\
Burn & 25 & $5.00(4.36)$ & $2-10$ \\
Scrape & 25 & $8.75(9.00)$ & $1-20$ \\
Insert & 18.8 & $11.5(12.02)$ & $3-20$ \\
Tattoo & 12.5 & $3.00(2.00)$ & $1-5$ \\
\hline
\end{tabular}

Note. All participants did at least one of the following: cutting, burning, or scraping. Participants reported first engaging in NSSI an average of 5.87 $(S D=4.03)$ years ago. NSSI = nonsuicidal self-injury; Cut = cutting the skin; Hit $=$ hitting the self (resulting in bruising or tissue damage $)$ B Burn = burning the skin; Scrape = scraping the skin; Insert $=$ inserting objects under the fingernails/skin; Tattoo = giving oneself a tattoo.

${ }^{a}$ Minimum-maximum. 


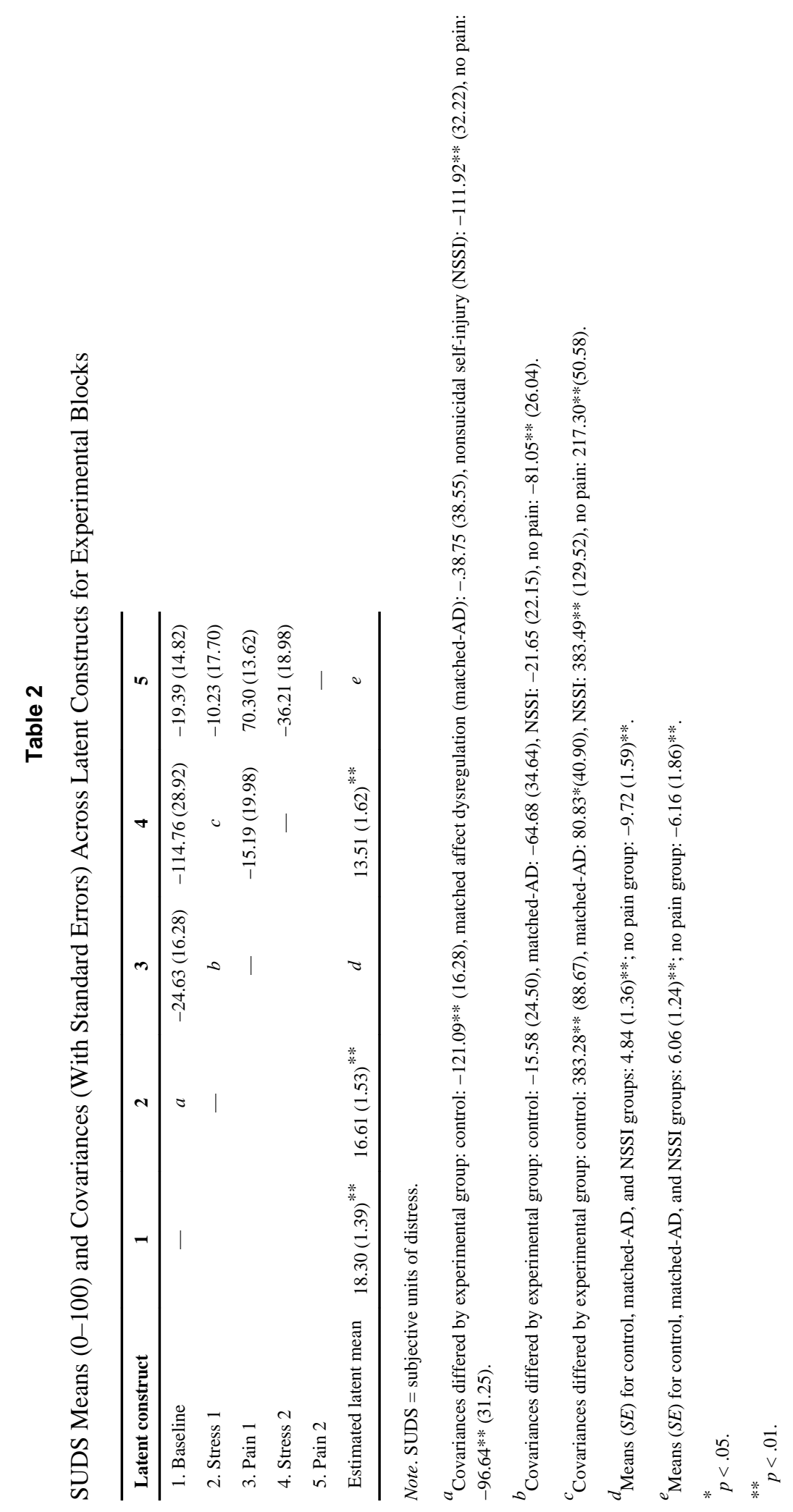




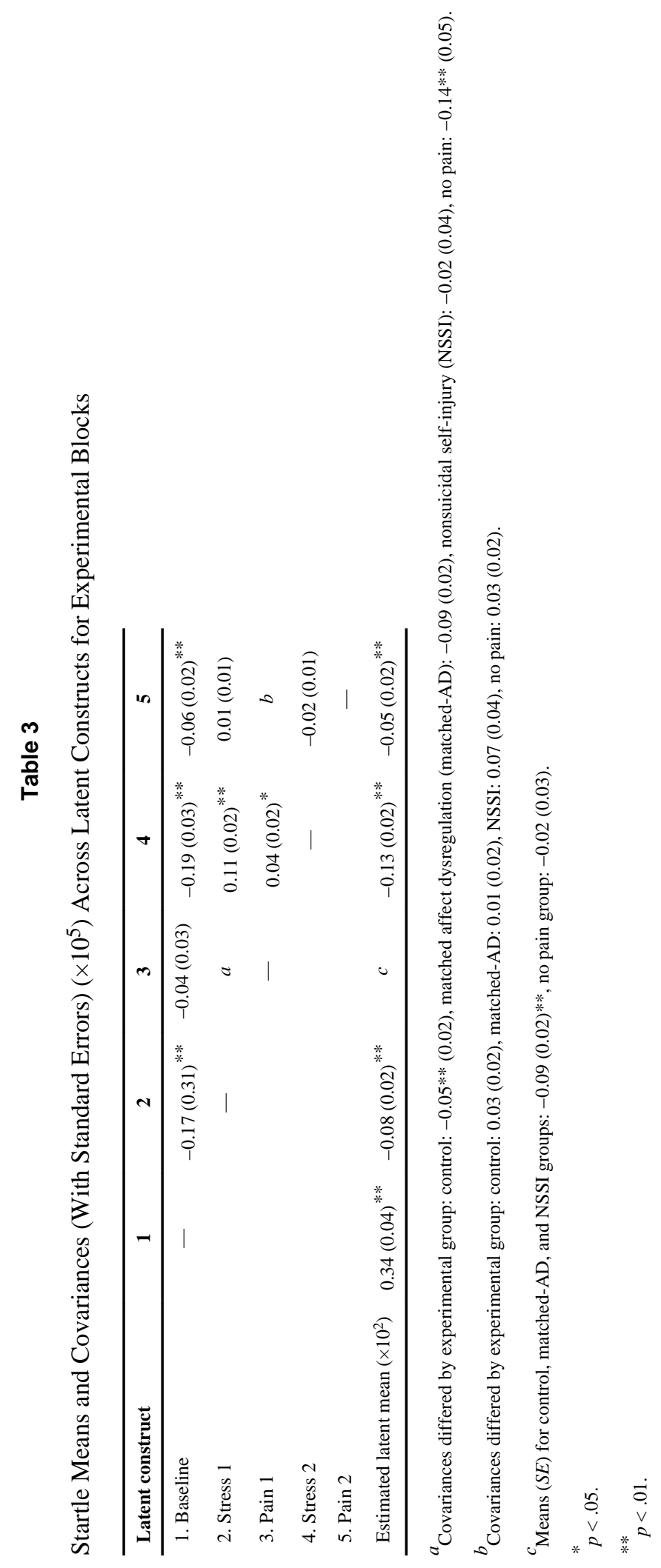




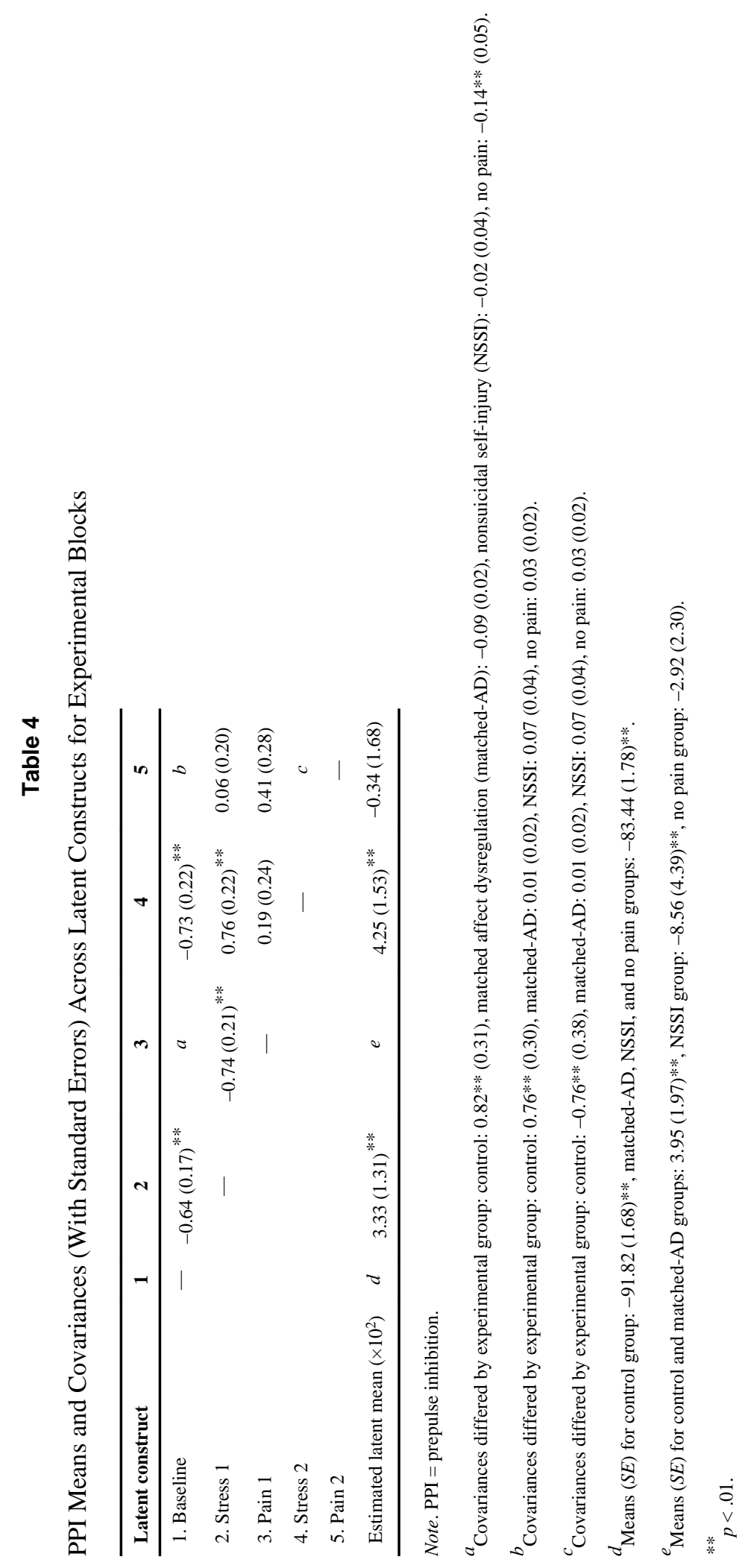

